\title{
Effects of an External Multi-Harmonic EMI Excitation on the Transmission Bit Error Rates of a Redundant Channel Under Plane Wave Illumination
}

\author{
Hassan Tirmizi \\ Dept. of Electrical Engineering \\ KU Leuven, Bruges Campus \\ Bruges, Belgium \\ hassan.tirmizi@kuleuven.be
}

\author{
Jonas Lannoo \\ Dept. of Electrical Engineering \\ KU Leuven, Bruges Campus \\ Bruges, Belgium \\ jonas.lannoo@kuleuven.be
}

\author{
Dries Vanoost \\ Dept. of Electrical Engineering \\ KU Leuven, Bruges Campus \\ Bruges, Belgium \\ dries.vanoost@kuleuven.be
}

\author{
Guy A. E. Vandenbosch \\ Dept. of Electrical Engineering \\ KU Leuven, Arenberg Campus \\ Leuven, Belgium \\ guy.vandenbosch@kuleuven.be
}

\author{
Davy Pissoort \\ Dept. of Electrical Engineering \\ KU Leuven, Bruges Campus \\ Bruges, Belgium \\ davy.pissoort@kuleuven.be
}

\begin{abstract}
In this paper, the effects of an external electromagnetic excitation comprising of various harmonics on a redundant communication channel are analysed and studied in detail. The geometry under study is subjected to strong incident plane waves with random angles of incidence and polarizations. The effects of a multi-harmonic Electromagnetic Interference (EMI) on the functional transmission signal of a redundant threechannel system is analyzed in a stochastic manner and the fault probabilities are quantified in terms of the resulting transmission Bit Error Rates (BERs).

The study shows that the harmonics of the external EMI have a strong influence on the system's functional response and lead to reduced immunity of the system which can lead to dangerous failures. The results reaffirm the need to realize and implement EMI resilience such that the system is inherently more robust against the adverse effects of electromagnetic disturbances and is able to cope with an uncertain harsh electromagnetic environment.
\end{abstract}

Index Terms-Electromagnetic Interference (EMI), EMI Resilience, Statistical Electromagnetics, Electromagnetic Compatibilty(EMC), Autonomous Systems

\section{INTRODUCTION}

Ours is a time of great technological breakthroughs and scientific discoveries. Within the last quarter of the past century, we have accomplished some magnificent scientific and engineering breakthroughs. These might soon lead to few other feats such as autonomous vehicles and smart-cities. These developments have been possible due to a technological revolution which has witnessed an ever increasing reliance

The research leading to these results has received funding from the European Union's Horizon 2020 research and innovation programme under the Marie Sklodowska-Curie Grant Agreement No 812.788 (MSCA-ETN SAS). This publication reflects only the author's view, exempting the European Union from any liability.

978-1-7821-5579-1/20/\$31.00 C2020 IEEE on small, highly interconnected electronic systems and wireless communication networks. However, these technological advancements have also been accompanied with fundamental changes in the electromagnetic environment. Fully electric cars are more susceptible to electromagnetic interference. Similarly, smart-grids with increasing number of renewable energy sources and non-linear power electronic converters cause more and more electromagnetic compatibility (EMC) and power quality problems. Furthermore, with advancements pertaining to Internet-of-Things (IOT) based cloud connectivity to achieve the vision of Industry 4.0, it is foreseen that the electromagnetic environment will become even harsher in the near future.

EMI immunity testing on its own is insufficient to test all the possible states in which a digital system can reside and can never cover all EMI disturbances which are likely to be encountered in an actual environment [1]. To cope up with the challenges associated with modern EMI environments and the shortcomings in the immunity testing related to the EMC directive, the topic of EMI Resilience has gained enormous traction recently [2]. The IEEE EMC Society's standard development project P1848 [3] enlists a set of EMI resilience techniques and measures which can help in realizing systems which are inherently more robust to EMI. One of the techniques is to use redundancy in conjunction with EM diversity in order to tackle the systematic and common cause effects of EMI [4]. Various successful implementations of EMI diverse systems, including time diversity [5], spatial diversity [6], inversion [7] and different matching schemes [8] have been reported in the literature.

In all aforementioned studies on EMI resilience, the predominant interference source comprised of a single frequency sinusoid. This paper goes further by studying the effects of multi- 
harmonic electromagnetic disturbances on the system under test. Accounting for the effects of harmonics and combined disturbances [1] has been identified as one of the problems associated with classical immunity testing. This study not only highlights the problems posed by disturbances composed of various harmonic frequencies but also provides a framework for implementing EM diversity techniques for embedding fault tolerance against EMI comprising of multiple frequencies.

The remainder of the paper is organized as mentioned ahead. In Section II, the simulation model is explained. Section III gives an overview about the calculation of induced voltages and how the transmission BERs are calculated. Section IV highlights the major results obtained from the study while section $\mathrm{V}$ lists some concluding remarks.

\section{Problem Formulation}

The analysis was carried out using an in-house built simulation framework in which the geometry, encoding schemes, EMI environment and the voting scheme can be freely selected. The following sections give an overview of the simulation set-up.

\section{A. Geometry Under Study}

The simulated geometry is shown in Fig 1. It consists of three redundant channels parallel to each other with equal spacing. The same geometry is chosen as in [5] where time diversity was studied for single frequency EMI. The three parallel micro-strips are driven by $1 \mathrm{~V}$ power supply with a source impedance of $50 \Omega$ and are terminated in a $50 \Omega$ load impedance.

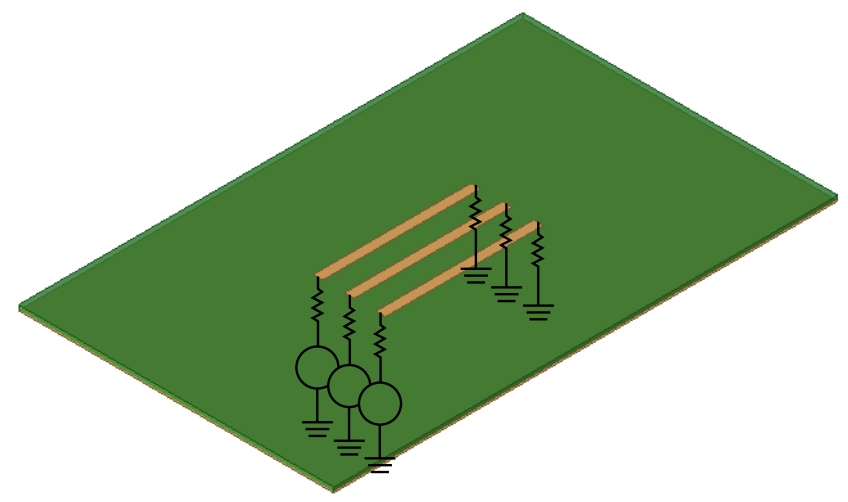

Fig. 1. Three Channel redundant system

Since the trace's characteristic impedance is matched to the load at the output port, the maximum output voltage for a 1 $\mathrm{V}$ applied signal is equal to $0.5 \mathrm{~V}$. The same bit stream is transmitted over the redundant channels as shown in Fig. 1 while the geometry at the same time is disturbed by EMI.

\section{B. Encoding Scheme}

A random stream of 100 bits is first encoded into voltages using a Non-Return-to-Zero-Level (NRZ-L) encoding scheme. A ' 0 ' bit is encoded as $0 \mathrm{~V}$ while a ' 1 ' is encoded as $1 \mathrm{~V}$.
The length of each bit is kept equal to the period of the bitfrequency $\left(f_{b i t}\right)$. EMI disturbances are sampled once per bit period. Fig. 2 shows a time domain visualization of a $200 \mathrm{MHz}$ EMI square wave disturbance superimposed on the encoded bit stream.

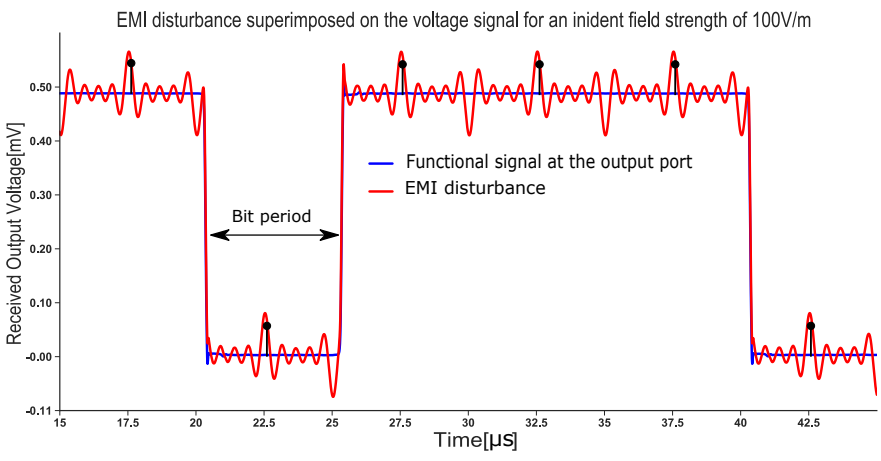

Fig. 2. EMI sampling at the middle of each transmitted bit

\section{Voting Scheme}

Redundancy along with majority voting is used in order to realize an EMI resilient system. So for a three-channel redundant system, a voting based on a '20o3' scheme is used to decide the voltage level at the output ports.

For decoding the voltages at the output ports, two thresholds are considered i.e. $1 / 3^{\text {rd }}$ and $2 / 3^{\text {rd }}$ of the maximum output voltage for a $1 \mathrm{~V}$ transmitted signal as shown in Fig. 3. Received voltages below $1 / 3 \quad V_{o}$ are decoded as '0' and above $2 / 3 V_{o}$ are decoded as ' 1 '. The received voltages in between these two thresholds are decoded as ' 2 ' and deemed as faulty bits, to consider a worst-case scenario.

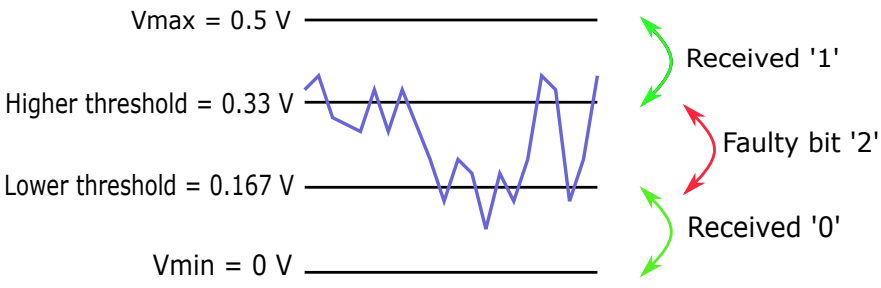

Fig. 3. Received voltage decoding at the output ports

The majority voter makes the final decision about which bit is actually received by looking at the three received decoded bits. The majority voter's outcome is the value that occurs two or three times among the three received decoded bits. Of course, it can and will happen that the majority voter's outcome is different from the actual transmitted bit. In this case, a bit error occurs as shown in Fig. 4. Finally, note that if any of the three received voltages falls in between both thresholds (the case of Faulty bit '2' in Fig. 3), a bit error is always assumed.

\section{EMI Environment}

Simultaneous EMI disturbances at different frequencies can occur in real-life scenarios. These are more likely to jeopardize 
system immunity as compared to the case where EMI testing is conducted for a discrete set of frequencies as independent events [1].

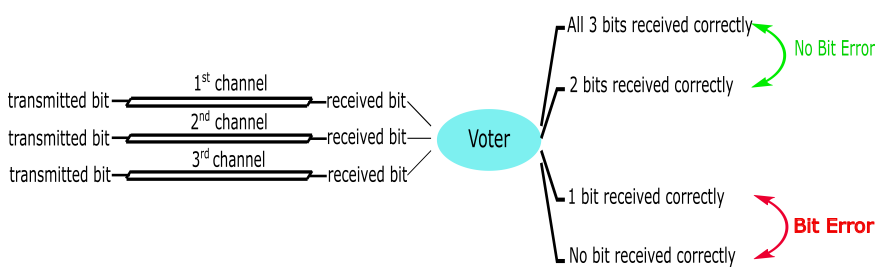

Fig. 4. '20o3' Majority voting scheme

EMI immunity testing doesn't take into account the vast range of scenarios i.e. the effect of harmonics, intermodulation, etc, in which multi-frequency disturbances can disrupt critical systems. Power electronic converters are a veritable source of electromagnetic pollution. With some tested specimens already using switching frequencies as high as $30 \mathrm{MHz}$ [9], the harmonics stretch way into the radiated emissions range. Fig. 5 shows the frequency spectrum of a typical square wave inverter when operated in the over modulation region.

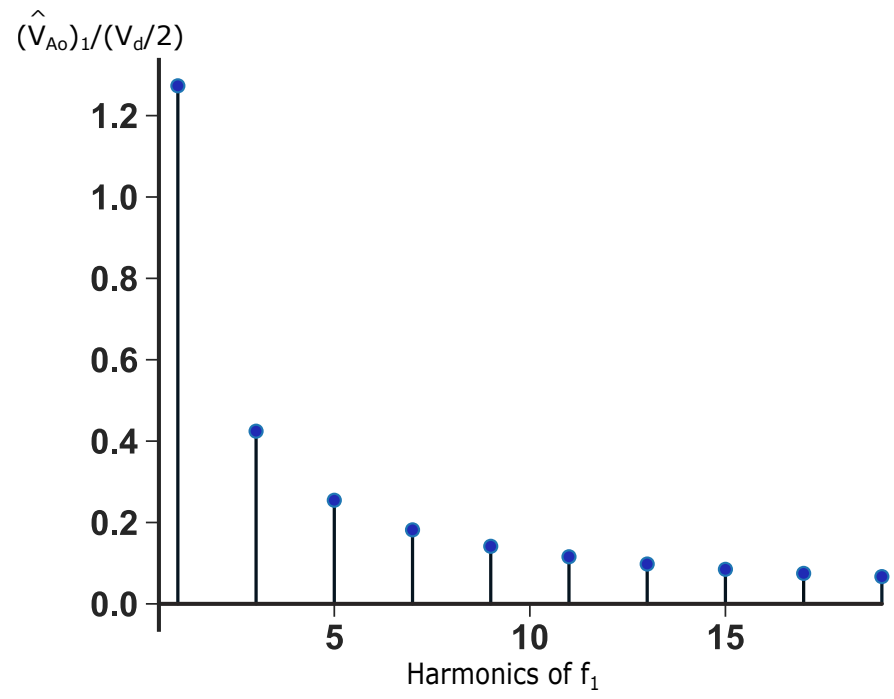

Fig. 5. Spectrum of a square wave inverter [10]

The EMI disturbance in this simulation model is chosen as a square-wave external excitation comprising of various harmonics of the fundamental frequency. To test the susceptibility of the system to harmonics of the fundamental frequency, a composite square waveform comprising of its first four odd harmonics is considered wherein all the harmonic components are in phase as shown in Fig. 6.

Unless explicitly mentioned otherwise,the fundamental frequency for the EMI excitation in all examples that follow is chosen as $200 \mathrm{MHz}$. Using the Fourier series representation, the square wave excitation can be written in terms of its harmonic components.

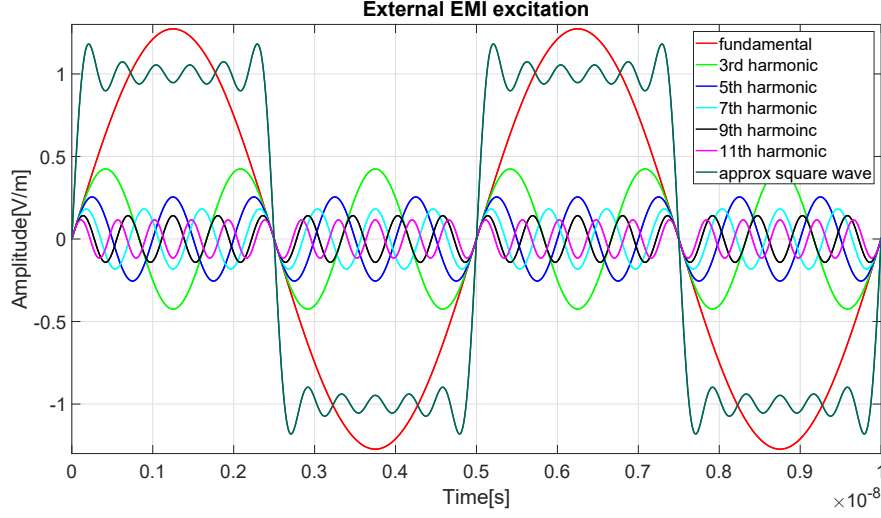

Fig. 6. A $200 \mathrm{MHz}$ squarewave EMI excitation along with its harmonic components

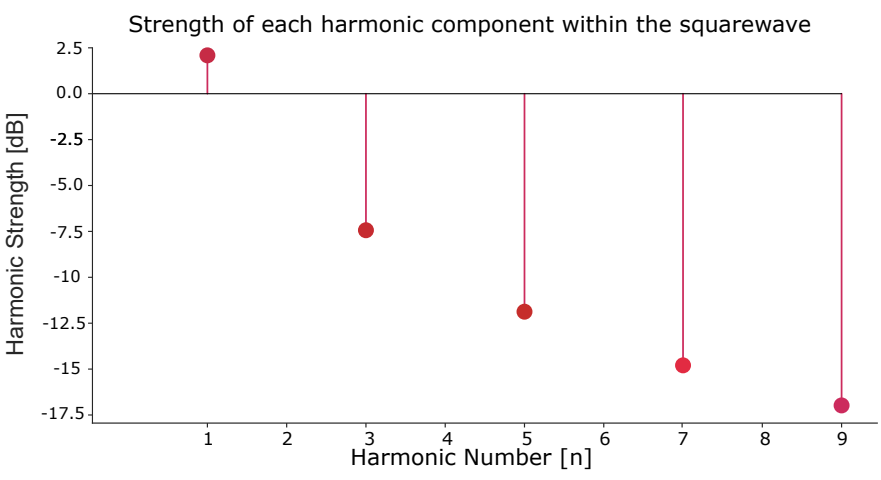

Fig. 7. Relative amplitude of the square wave EMI harmonics

$$
E(t)=a_{o}+\sum_{n=1}^{\infty} a_{n} \cos \left(n \omega_{n} t\right)+\sum_{n=1}^{\infty} b_{n} \sin \left(n \omega_{n} t\right)
$$

where

$$
\begin{gathered}
a_{o}=0 \\
a_{n}=0 \\
b_{n}=\frac{4 A(1-\cos (n \pi / 2))}{n \pi}
\end{gathered}
$$

$E(t)$ represents the external EMI excitation, $a_{o}$ represents the average value while $a_{n}$ and $b_{n}$ represent the even and odd fourier coefficients respectively, and $n$ represents the harmonic number. Since the waveform is half-wave symmetric, only odd harmonics i.e. $b_{n}$ 's exist. The magnitude of the harmonics decreases as the harmonic number increases. Therefore, for the analysis, only those harmonics are considered where the relative amplitude of the harmonic strength and the squarewave amplitude is at least $-20 \mathrm{~dB}$ or more as shown in Fig. 7.

\section{Calculation of Induced Voltages}

To calculate the induced voltages on the redundant threechannel system in an efficient manner, the Antenna Reciprocity theorem is used [11]. The geometry's far-field response in the 
transmit mode is captured for each port and each harmonic frequency with a single full wave simulation. The far-field patterns along with the characteristics of the particular EMI waveform are then used to calculate the received voltage at the ports in the time domain. The magnitude of the recorded electric far field patterns are appropriately scaled to account for the strength of each individual harmonic component within the square wave.

$$
E_{f f_{n}}(\theta, \phi, \psi)=\frac{4}{n \pi} \cdot\left|E_{f f}\left(f_{n}, \theta, \phi, \psi\right)\right|
$$

where $f_{n}$ represents the frequency, $E_{f f}$ represents the electric far-field and $E_{f f_{n}}$ represents the far-field response of the $n^{t h}$ frequency component.

To check the correctness and accuracy of our antenna reciprocity theorem implementation, the received voltage at the port terminals of the redundant system has been verified for various angles of incidence by comparing the results with those obtained with the simulation in the FDTD Solver of EMPro by Keysight Technologies. Fig. 8 shows the comparison for a particular angle of incidence.

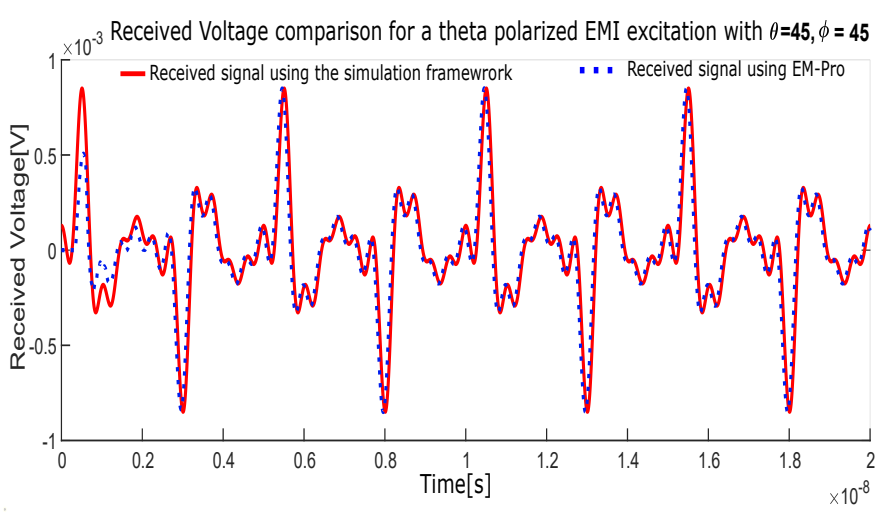

Fig. 8. Received Voltage comparison between EMPro and the Simulation framework

As shown in Fig. 8, the actual induced voltage at the port terminals is no longer a square wave, despite the fact that the EMI disturbance was a square wave. A Fourier decomposition of the received voltage as shown in Fig. 9 gives a better insight of this. The individual frequency components undergo a change in their magnitude as well as phase such that the combined composite waveform at the ports is no more a square wave.

\section{Bit Error Rate Calculation}

To calculate the BER, the external multi-frequency EMI (planewave) is swept over all possible angles of incidence, polarizations and phases with which it can impinge on the device under test (DUT). As shown in Fig. 10, the angles $\theta, \phi$ and $\psi$ determine the spatial orientation of the incoming disturbance waveform while the phase angle $\alpha$ accounts for the time delay that occurs in the excitation waveform while travelling from the source towards the system under test.

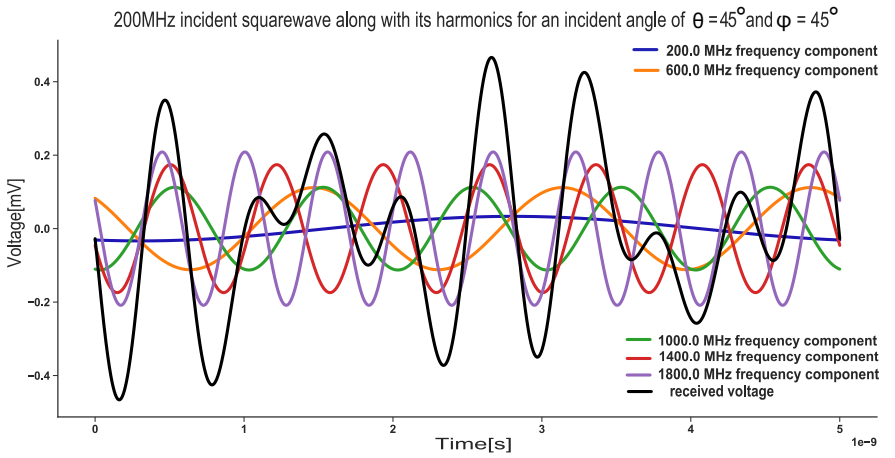

Fig. 9. Received port voltage along with its harmonic frequency components

Since the medium of propagation of the travelling wave is air, all the harmonic frequency components undergo the same phase delays. However, due to frequency-dependent group delays, the relative phase between the harmonic components is no more zero. Furthermore, the system's frequency response decides which harmonic components get suppressed and which ones get amplified. Fig. 9 illustrates this point by showing the output response of a theta polarized EMI square wave signal with both the polar and azimuthal angles of incidence chosen as $45^{\circ}$.

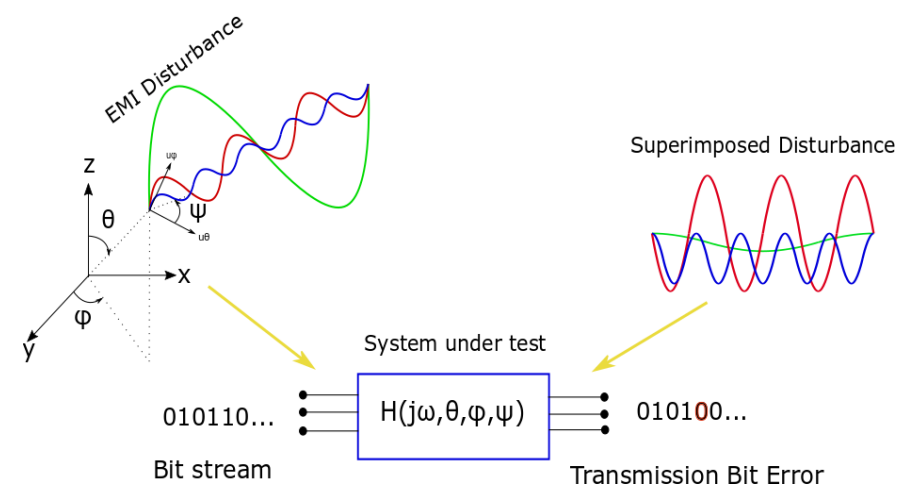

Fig. 10. Received port voltage along with its harmonic frequency components

The BER is calculated using the following formula.

$$
\mathrm{BER}=\frac{\text { number of bits received incorrectly }}{\text { bitpattern length } . n_{\theta s} \cdot n_{\phi s} \cdot n_{\psi s} \cdot n_{\alpha s}}
$$

where

$n_{\theta s}$ represents the number of polar angles.

$n_{\phi s}$ represents the number of azimuthal angles.

$n_{\psi s}$ represents the number of polarization angles.

$n_{\alpha s}$ represents the number of time delays considered.

\section{RESULTS}

\section{A. Comparison within the Square Wave Harmonic Compo-} nents

In a first simulation, BERs were computed as a function of the square-wave incident field strength for each individual 
harmonic frequency present within the square wave. In Fig.11, the total BER and the fault probabilities associated with each type of fault is compared for the fundamental and the $3^{\text {rd }}$ harmonic component as a function of the field strength. It is worth noticing that in addition to the BER curve which shifts towards the left for the $3^{\text {rd }}$ harmonic component, the fault probability for the three trace fault scenario indicated by the red region in the stack plot also increases. The occurrence of a three trace fault is a potentially dangerous scenario for safety-critical systems. Since the transmitted bits on all the three channels undergo a flip and thereby the voter is not able to detect the presence of an EMI event.

In Fig. 12, the effect of the fundamental and the first three odd harmonics of a $200 \mathrm{MHz}$ fundamental frequency excitation in terms of the resulting BERs can be seen. It is worth noting that the $3^{\text {rd }}$ and the $5^{\text {th }}$ harmonics, despite their significantly reduced amplitudes compared to the fundamental component, introduce higher BERs.
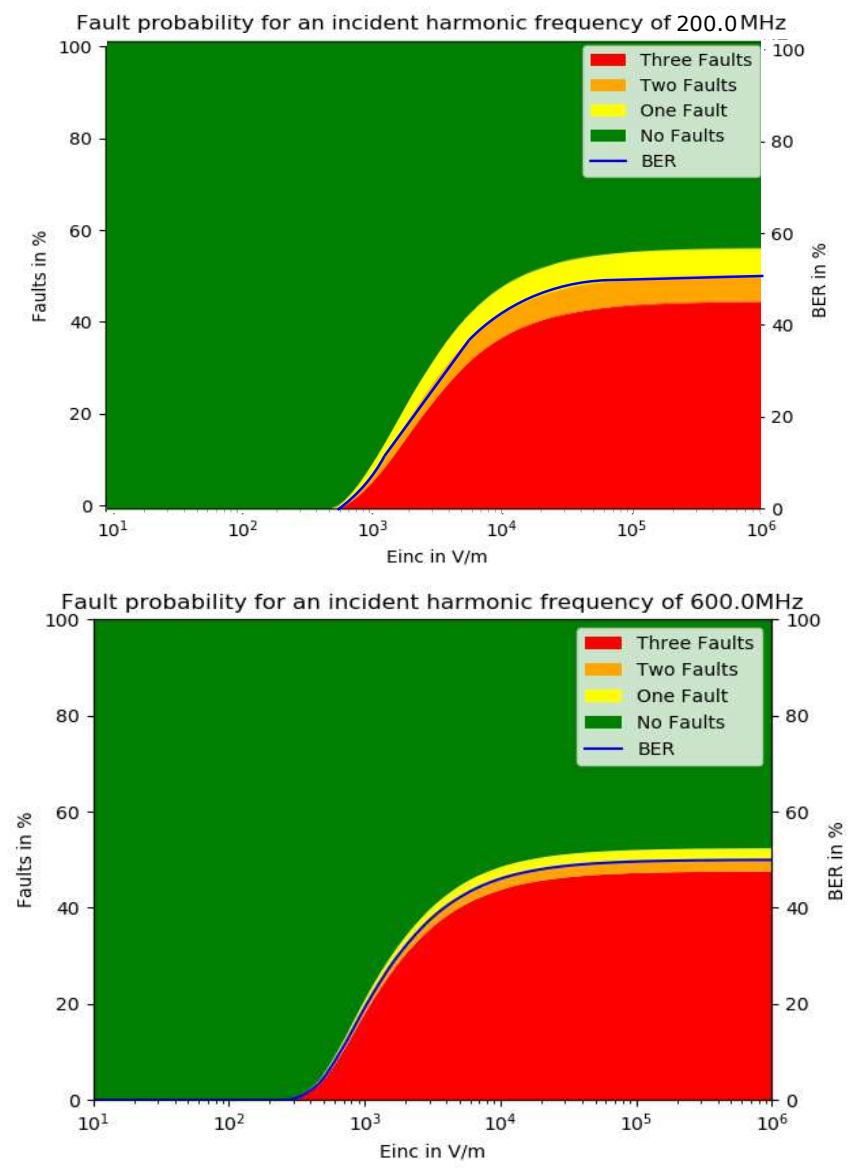

Fig. 11. Fault probability comparison between the fundamental and the $3^{\text {rd }}$ harmonic frequency component

\section{B. Comparison between the Single Frequency Case and a Square Wave}

In a second simulation, we compared the composite square wave with the single frequency sinusoid case for plane

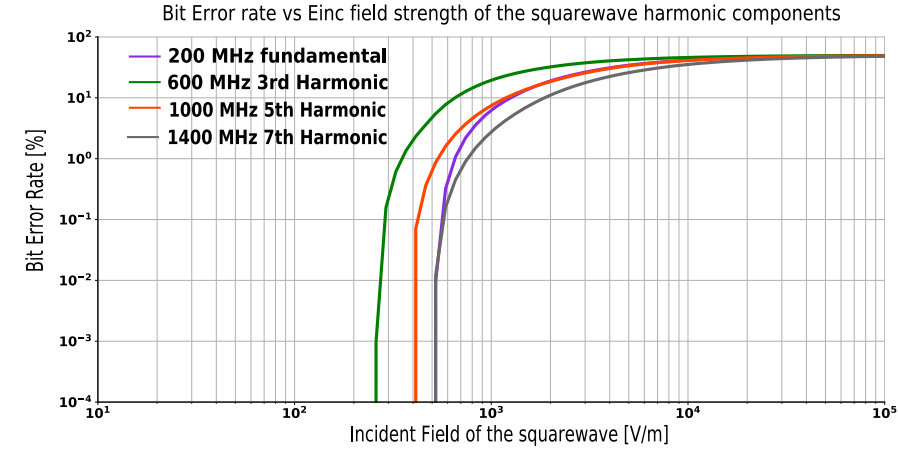

Fig. 12. BER comparison for individual harmonic components within the $200 \mathrm{MHz}$ square wave

wave conditions. Fig. 13 shows that due to a square wave disturbance, the system immunity has worsened a lot in comparison to the case where only single frequency sinusoids are considered. The BER due to a combined square wave is not simply a linear combination of the BERs induced owing to the individual harmonic components present in the square wave.

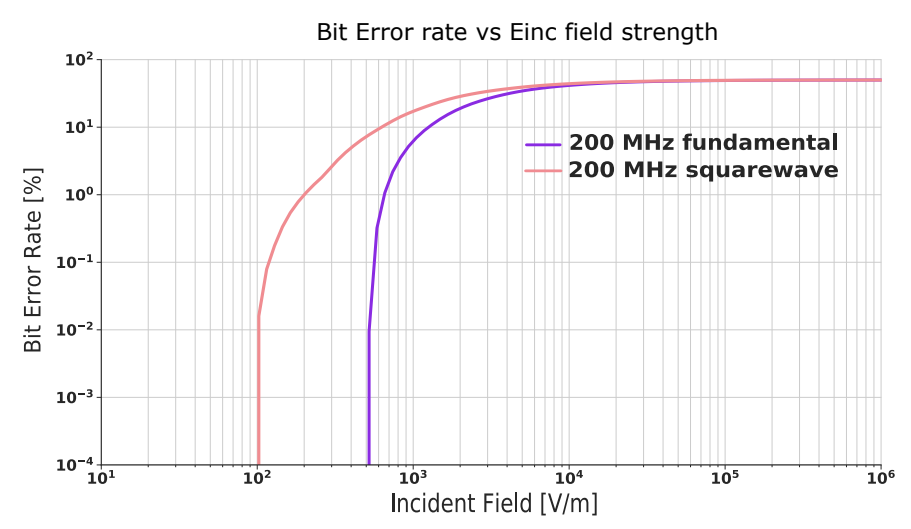

Fig. 13. BER comparison for a sinewave and a squarewave disturbance with equal amplitude

A step further was taken by computing the BER as a function of the fundamental frequency of the square wave and comparing it with the sinusoidal scenario. For this simulation, a frequency range of 0 to $1 \mathrm{GHz}$ was chosen with a resolution of $25 \mathrm{MHz}$. As shown in the red curve of Fig.14, each BER value corresponds to a square-wave which in turn also has contribution from its first four odd harmonics.

Further, it can be noticed that there is negligible contribution to the BERs for the single frequency case for frequencies below $200 \mathrm{MHz}$ whereas for the square wave case BER takes a non-zero value already starting from $25 \mathrm{MHz}$ onwards. This fact is also worth noting that both BER curves reach a plateau around the frequency of $600 \mathrm{MHz}$. This can possibly be due to the resonant frequency of the system under study and is worth exploring. 


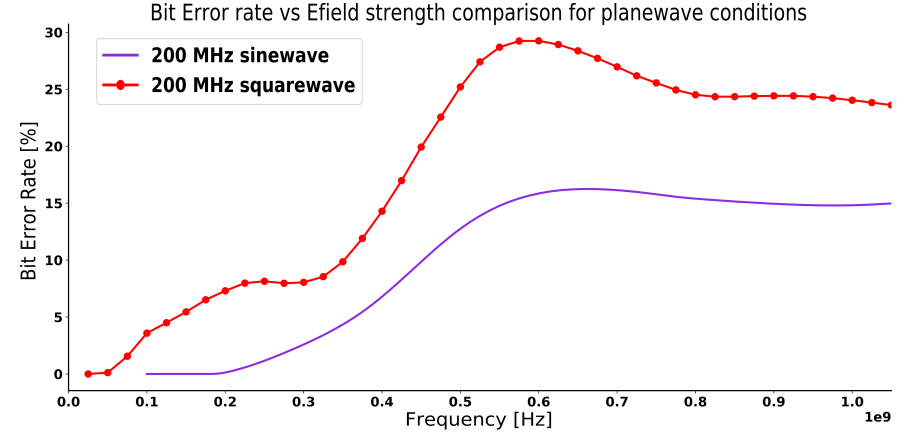

Fig. 14. Comparison of BER as a function of the frequency for a sine wave and square wave disturbance with an E-field strength of $500 \mathrm{~V} / \mathrm{m}$

\section{CONCLUSIONS}

A detailed study was undertaken to investigate the effects of concurrent multiple EMI disturbances on redundant communication channels in plane wave conditions. As a particular case, a square-wave EMI disturbance was simulated using an inhouse built simulation framework. The results show a clear degradation of performance and reduced immunity in comparison to the single frequency excitation case.

As future work, it would be interesting to model multiple disturbances in a reverberation room environment to take into account the effect of reflections on system immunity. It would also be worthwhile to investigate the performance of various other types of EM-diverse systems for multiple frequency disturbances in harsh EMI environments.

\section{REFERENCES}

[1] K. Armstrong, "Why increasing immunity test levels is not sufficient for high-reliability and critical equipment," in 2009 IEEE International Symposium on Electromagnetic Compatibility. IEEE, 2009, pp. 30-35.

[2] D. Pissoort and K. Armstrong, "Why is the ieee developing a standard on managing risks due to em disturbances?" in 2016 IEEE International Symposium on Electromagnetic Compatibility (EMC). IEEE, 2016, pp. 78-83.

[3] D. Pissoort, J. Lannoo, J. Van Waes, A. Degraeve, and J. Boydens, "Techniques and measures to achieve emi resilience in mission-or safetycritical systems," IEEE Electromagnetic Compatibility Magazine, vol. 6, no. 4, pp. 107-114, 2017.

[4] K. Armstrong, "Techniques and measures to manage functional safety and other risks with regard to electromagnetic disturbances," in 2018 IEEE 4th Global Electromagnetic Compatibility Conference (GEMCCON). IEEE, 2018, pp. 1-5.

[5] J. Lannoo, J. Van Waes, A. Degraeve, D. Vanoost, J. Boydens, and D. Pissoort, "Effectiveness of time diversity to obtain emi-diverse redundant systems," in 2018 International Symposium on Electromagnetic Compatibility (EMC EUROPE). IEEE, 2018, pp. 288-292.

[6] A. Degraeve and D. Pissoort, "Study of the effectiveness of spatially em-diverse redundant systems under plane-wave illumination," in 2016 Asia-Pacific International Symposium on Electromagnetic Compatibility (APEMC), vol. 1. IEEE, 2016, pp. 211-213.

[7] J. Lannoo, A. Degraeve, D. Vanoost, J. Boydens, and D. Pissoort, "Effectiveness of inversion diversity to cope with emi within a twochannel redundant system," in 2018 IEEE International Symposium on Electromagnetic Compatibility and 2018 IEEE Asia-Pacific Symposium on Electromagnetic Compatibility (EMC/APEMC). IEEE, 2018, pp. $216-220$.

[8] — " "Study on the use of different transmission line termination strategies to obtain emi-diverse redundant systems," in 2018 IEEE International Symposium on Electromagnetic Compatibility and 2018 IEEE Asia-Pacific Symposium on Electromagnetic Compatibility (EMC/APEMC). IEEE, 2018, pp. 210-215.
[9] X. Ke, J. Sankman, M. K. Song, P. Forghani, and D. B. Ma, "16.8 a 3 to-40v 10-to-30mhz automotive-use gan driver with active bst balancing and vsw dual-edge dead-time modulation achieving $8.3 \%$ efficiency improvement and 3.4 ns constant propagation delay," in 2016 IEEE International Solid-State Circuits Conference (ISSCC). IEEE, 2016, pp. 302-304.

[10] N. Mohan, T. M. Undeland, and W. P. Robbins, Power electronics: converters, applications, and design. John wiley \& sons, 2003.

[11] F. Vanhee, D. Pissoort, J. Catrysse, G. A. Vandenbosch, and G. G. Gielen, "Efficient reciprocity-based algorithm to predict worst case induced disturbances on multiconductor transmission lines due to incoming plane waves," IEEE Transactions on Electromagnetic Compatibility, vol. 55, no. 1, pp. 208-216, 2012. 